\title{
The embarrassment of riches: 'Head' words in the Indo-European family
}

\author{
Piotr Gąsiorowski \\ Faculty of English, Adam Mickiewicz University in Poznań \\ gpiotr@wa.amu.edu.pl
}

\begin{abstract}
The notion of HEAD is reflected in the basic lexicon of all known languages; the identification of the head as a distinct and vitally important body part, labelled with a simplex word, seems to be a cross-cultural universal. Thanks to their high frequency of use and their "basic concept" status, words meaning 'head' tend to be diachronically stable and therefore important for comparative reconstruction. Their expected retention rate - as estimated on the basis of data from several uncontroversial language families - is on a par with words meaning 'heart' or 'foot'. On the other hand, culture-specific factors may lead to the proliferation of secondary meanings, the rise of stylistically marked nearsynonyms, and consequently to locally accelerated lexical evolution. This seems to have happened repeatedly in the Indo-European family, in which not only the oldest reconstructible 'head' word, *kreh ${ }_{2}$ - but also secondary, branch-specific terms have often been subject to lexical replacement. This unusual variability of words for HEAD in IndoEuropean contrasts with the remarkable conservatism of words for several other bodypart concepts, such as EYE, EAR, TOOTH and HEART. In this paper, we shall attempt to identify recurrent patterns of semantic change in the emergence of new synonyms and the polysemic development of inherited 'head' words. Insights derived from recent studies of "embodiment" will be used to explain the observed tendencies.
\end{abstract}

\section{Introduction ${ }^{1}$}

The head is one of the principal anatomical components not only in humans but also in most other animals. Its significance as a distinct and vitally important body part is reflected in linguistic usage. The meaning 'head' is included in most standard checklists of "basic concepts", such as the Swadesh list. This

\footnotetext{
${ }^{1}$ I am grateful to two anonymous reviewers of the Yearbook of the Poznan Linguistic Meeting for their helpful remarks on an earlier draft of this article. I have done my best to fix the weaknesses they pointed out and expand the argument where necessary. All remaining flaws are my responsibility alone.
} 
concept seems to be labelled with simplex primary lexemes in all known languages, as noted already by Brown (1976: 405) and Andersen (1978: 352-353); the latter explicitly elevates this observation to the status of a cross-cultural lexical universal. ${ }^{2}$ Goddard (2001: 57) includes 'head' in his very short list of "[t]he best candidates for the status of universal meanings".

Thanks to their high frequency of use ${ }^{3}$ and their "basic" character, words meaning 'head' can be expected to be diachronically conserved - that is, highly resistant to lexical replacement. ${ }^{4}$ This expectation is borne out by the evidence: the average retention rate of words for 'head' (over a large number of uncontroversial language families) is on a par with other frequently used terms for internal or external body parts, such as 'heart' or 'foot' (Holman et al. 2008: 351). For example, Proto-Austronesian *qulu 'head' can be securely reconstructed on comparative grounds. Its historical reflexes are widely distributed in the Austronesian family. They generally retain the core sense of 'head', though many Austronesian languages extend it metaphorically to secondary senses such as 'top/summit; chief/leader; handle (of knife, etc.); upper course of river; prow of a boat; first-born child; earlier, before' (Blust 2013: 322-323). Given the immense size and geographical range of Austronesian, its time-depth (of ca. 6000 years) and complicated history, the stability of this particular form-to-meaning mapping is impressive indeed. Such stability, however, is not constant across language families, and word frequency is not the only factor that determines lexical replacement rates. ${ }^{5}$ There are cases when lexical and semantic evolution seems to accelerate, selectively reducing the life expectancy of certain highfrequency words. We shall try to identify some of the factors that play a role in precipitating such shifts.

\section{2. 'Head' in Proto-Indo-European}

The Indo-European (IE) languages also preserve a large number of lexical archaisms denoting body parts or organs - including, among others, the reflexes

\footnotetext{
${ }^{2}$ See also Enfield's (2006: 148-152) guide for field linguists who collect terms for body parts, where the "basic" status of HEAD as a cognitive category is taken for granted.

${ }^{3}$ For example, the noun head ranks 252nd on the word-frequency list derived from the Corpus of Contemporary American English (http://www.wordfrequency.info/free.asp, retrieved on 1 February 2017). It occurs ca. 356 times per million words in typical contemporary English usage.

${ }^{4}$ For a classic demonstration that word frequency alone is a strong predictor of the lexical replacement rate, see Pagel, Atkinson and Meade (2007).

${ }^{5}$ See, recently, Vejdemo and Hörberg (2016), who estimate the effect of several semantic factors.
} 
of such items as $* h_{3} o k^{w_{-}}$'eye', *háuses- 'ear', *h$h_{1} d o n t-/ * h_{1} d n t-$ 'tooth',

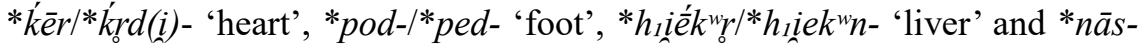
/*nas- 'nose'. Their old age is warranted not only by their distribution in the branches of the family, but also by their formal traits. These nouns are consonantal stems, for the most part morphologically opaque and synchronically unanalysable already in Proto-Indo-European (PIE); their declension often involves morphophonological oddities such as recessive ablaut patterns and stem heteroclisy. It may come as a surprise that some common words for body parts in IE seem to behave differently from the rest of this conservative package. These include, notably, the words for 'hand' and 'head'. The presence of cognate forms in Hittite, Tocharian, Greek, Armenian and Albanian allows us to reconstruct PIE * $g^{\prime} e^{\prime} s \bar{o} r /{ }^{*} g^{h}$ esr- 'hand' quite confidently, but its reflexes have a much more restricted distribution than those of the 'foot' word. ${ }^{6}$ We see a variety of lexical innovations replacing the inherited term for 'hand' in Italic, Celtic, Germanic, Balto-Slavic and Indo-Iranian. As will be shown, the case of 'head' is even more erratic.

The evidence for a PIE word labelling the concept of 'head' is so meagre that we have to rely on internal reconstruction and circumstantial reasoning to recover its approximate form. It is only in two branches of IE - Greek and Indo-Iranian - that we find cognates meaning 'head': Ancient Greek kárā, Vedic śíras- (gen. sg. śìrșnás), and Younger Avestan sarah-. The Greek word can mean not only 'head' (human or animal), but also synecdochically 'person' or metaphorically 'peak'. It functions as a poetic synonym of the usual 'head' word, kephalế, rather than a primary anatomical term. Vedic síras- is similarly polysemous, with such typical senses as 'head, skull', 'peak, top, upper end' and 'leader, chief'; it is however the stylistically unmarked, default word for 'head' in the language, unlike its Greek cognate. In other branches the meaning 'head' is not attested, though we have a probable trace of the word in the Hittite adverb ketkar 'at the head', presumably a fossilised prepositional phrase similar to the Homeric hapax legomenon epi kár 'headlong' (Iliad 16: 392). The PIE state of affairs is unclear. We can agree with Nussbaum (1986: 103) that there must have been a PIE neuter substantive with the core meaning of 'head', containing the root morpheme * $k^{\prime}(e) r$ - and the suffix $*_{-}(e) h_{2^{-}}$. Whether we should accept the analysis favoured by Nuss-

\footnotetext{
${ }^{6}$ It seems that, as in many other languages worldwide, the PIE primary lexemes conventionally glossed 'foot' and 'hand' could actually be used to refer to the whole limb: 'leg (and foot)' and 'arm (and hand)', respectively. This is still the case in some modern IE languages (see the discussion in Brown 1976: 405-408).
} 
baum (1986: 100: $\left.* k^{\prime} r-e h_{2} /{ }^{*} k_{0}^{\prime}-h_{2}-\right)$ is less certain, given the limited evidence. In Indo-Iranian the word is further extended into an $(e) s$-neuter (as if reflecting earlier $* k^{\prime} h_{2}-O s$ ) with heteroclitic, $n$-final weak cases attested in Vedic (gen. $* k r h_{2}-s-n$-és etc.). It is hard to tell how old these forms are: $n$-heteroclisy was common in the semantic class of body parts and could spread analogically in post-PIE times, producing "dialectal" variants.

Although the PIE 'head' lexeme has left so few direct reflexes, several words derived from it have survived in various parts of the IE family with highly modified meanings. They testify to its highly active role as a derivational basis. ${ }^{7}$ We have, for example, Latin cerebrum 'brain' < *kerh ${ }_{2}-s-r o-$ and crābrō 'hornet' $<* k_{0}^{\prime} h_{2}-s-r o-\left(h_{3}\right)$ on- ${ }^{8}$ The details of their morphological and semantic evolution are exhaustively discussed in Nussbaum's (1986) monographic treatment of this word-family and there is no need to revisit them here; let us only note the paradoxical fact that the IE 'hornet' word, found also in Germanic and Balto-Slavic, ${ }^{9}$ has fared rather well in a few IE lineages, all of which lost the original 'head' word already in prehistoric times. In contemporary English usage, hornet (itself a distant relative of $c r a \bar{a} b r \bar{o}$ ) is three orders of magnitude less frequent than head.

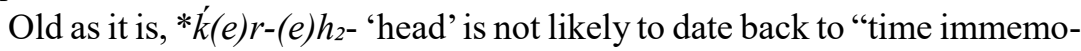
rial" beyond the reach of etymology. It is derivable, within PIE, from the morpheme *ker-, which underlies a large word-family built around the meaning 'horn' (material) or 'horn, antler' (of an animal). ${ }^{10}$ Nussbaum argues that the meaning 'head' developed via such intermediary steps as 'head-bone' (substance) $\rightarrow$ 'skull' (object) > 'head'. This would mean that the PIE 'head' word was, on an average, relatively short-lived. It was formed in the last common ancestor of the family and lost early in most of the daughter languages, except the

\footnotetext{
${ }^{7}$ Another case in which an anatomical term was lost at an early date leaving behind a large wordfamily is provided by the PIE root noun * $h_{2}$ ant- 'forehead', preserved with this meaning only in Anatolian (Hittite hant-) but well represented throughout IE by grammaticalised case forms (loc. sg. * $h_{2} a ́ n t-i>$ Lat. ante 'before, in front', Greek anti 'against, opposite', Vedic ánti 'facing') and derived lexemes (e.g. English end < Proto-Germanic *anðijaz, cf. Ancient Greek antios 'set against, facing').

${ }^{8}$ Cf. also Ancient Greek krānion 'brainpan, skull', German Hirn 'brain', Old Russian sbršenb 'hornet', etc.

${ }^{9}$ Cf. also Finnish herhiläinen, an early loan from Baltic.

${ }^{10} \mathrm{Cf}$. Latin cornū, Proto-Germanic *xurna- (English horn), Greek kéras, Vedic śŕnga-, Hittite karāwar, etc.
} 
Indo-Iranian branch, where its reflexes have survived till present with the same core meaning. ${ }^{11}$

\section{Branch-specific innovations}

Several branches of IE have their own words for 'head'. ${ }^{12}$ Some of them have transparent etymologies, whereas others look enigmatic. Slavic and Baltic share the same word, Proto-Balto-Slavic *galw $\bar{a}^{2}$ (Old Church Slavonic glava, Lithuanian galvà, etc.). It seems to be related to Slavic *golı 'bare, naked' and Germanic *kalwa- 'bald', so we may be dealing with a semantic shift from 'bald head, skull' to 'head'. Old Armenian glux 'head' is often regarded as related to the Balto-Slavic word, but the comparison is entirely speculative, based on the superficial similarity of the words and not supported by a careful etymological analysis.

Latin has caput (gen. sg. capitis), a consonantal neuter stem. Again, its etymology is uncertain, but if we analyse it as *kap-ut-, it can be interpreted as a derivative of the verb root *kap- 'seize, grasp' (as in Latin capio 'take'). This root occurs in words that refer to various types of containers in several IE languages, e.g. Latin capsa 'box' and Celtic *kapuko- 'drinking-cup, bowl'. As we shall see, the semantic development 'vessel, container' > 'head' is common cross-linguistically. Germanic has a word for 'head' whose similarity to caput cannot be accidental, but which exhibits puzzling variation across Germanic languages. The North Germanic variant (Old Icelandic hofuð) points to a preform like *xafuða- or *xaßuða-, which matches the Latin word almost perfectly (except that the Germanic stem is thematic). The Gothic and West Germanic forms, however, go back to *xaußiða- ${ }^{13}$ Outgroup comparison suggests that the

\footnotetext{
${ }^{11}$ Cf. Modern Persian sar, Ossetian scer, Punjabi sir, European Romani šero, etc.

${ }^{12}$ As one of the reviewers has kindly reminded me, a still useful though incomplete overview of the words for 'head' in a number of IE languages, with a brief discussion of their etymologies and transferred meanings, can be found in Buck (1949: 212-213). The entry for 'head' is immediately followed by several related subentries, including those for 'skull' and 'brain' (Buck 1949: 213 215). Of course IE studies have progressed greatly in the past decades, supplementing or invalidating some of the research on which Buck's dictionary was based, but his work, if handled with care, remains a convenient starting-point for studies of semantic evolution in the IE family.

${ }^{13}$ Except Old English heafod $<$ pre-OE *haußud, which may owe its *u either to assimilatory vowel retraction (generalised from its plural forms, which all had a back vowel in the final syllable) or to the analogy of the variant *hafud $<* x a f u d a-$, which is marginally attested as a compositional element in Old English hafudlond 'headland' (a strip of land at the edge of a planted field).
} 
North Germanic form is more conservative, but the distribution of the variants inside Germanic clearly indicates that *xaußiða- must have existed already in Proto-Germanic. To complicate matters further, we also have the Old English $n$ stem synonym hafela 'head' < *xafulan-, not strictly cognate but possibly containing the same root; it could even have originated as a diminutive of * xafuða-. It seems, therefore, that the variation *xafuða- *xaußiða- should be projected back onto Proto-Germanic. It may reflect a pre-Germanic neuter paradigm with mobile accent and suffix ablaut, like *káp-ut/*kap-uét-, with metathesis in the oblique form to avoid a dispreferred cluster $[\mathrm{pw}],{ }^{14}$ with two contiguous labials, in the onset of the second syllable. Tentative as this explanation is, it allows us to reconcile the Latin and Germanic evidence and posit a "dialectal" IE word shared by Italic and Germanic.

Celtic * $k^{w}$ ennom 'head' (Gaulish penno-, Old Welsh penn, Old Irish cenn) is both enigmatic and unique to that branch. The word has no obvious external cognates and no Indo-European etymology. We do not know what its original meaning was (if not 'head'). Given the outstanding symbolic importance of the head in Celtic religion and culture, the evidence of head cults and head-hunting, and the ubiquity of severed heads, skulls and ritual skull cups as motifs in Celtic literatures and iconography, there may have been a strong cultural incentive for the spread of a new word for 'head'. Borrowing from a non-Indo-European source as a result of external cultural impact is possible, though neither demonstrable nor falsifiable at present.

Albanian kokë 'head' is thought to reflect Vulgar Latin coccum 'berry', itself a loanword from Greek kókkos, which could refer to pomegranate seeds or to Kermes vermilio - a berry-shaped insect used to make a crimson dye. The Latin borrowing has taken over the core meaning of krye, an indirect reflex of the PIE 'head' word and a close cognate of Ancient Greek krānion 'brainpan, skull'. While giving up its old primary sense, Modern Albanian krye has retained a great wealth of secondary ones: 'chief, boss', 'head (counting unit) of cattle or sheep', 'beginning or end, starting-point, source', 'chapter' etc.; it is also common as the first member of compounds, where it means 'arch-, main, chief'.

Ancient Greek kephalê, the usual word for 'head' in the language, has cognates in Tocharian A (śpāl 'head') and Germanic (Gothic gibla, OHG gibil, gebal <*yeßla(n)-, *yißila- 'the highest point, pinnacle'). The word is surely old in Indo-European and may have served as a secondary term for 'head' at an early stage in the history of the family. Judging from the position of Tocharian in

${ }^{14} \mathrm{Or}[\beta \mathrm{w}]$, if the metathesis happened in Proto-Germanic proper, after the operation of Grimm's and Verner's Laws. 
the IE family tree (as a sister taxon to the "crown group" containing all the extant IE languages), the meaning 'head' may even be older than those found in Germanic. Verb roots of the shape * $g^{h} e b^{h} \sim^{*} g^{h} a b^{h}$ occur in several branches of IE with meanings such as 'give', 'seize, take' or 'have, hold', and thus semantically close to the *kap-root mentioned in connection with Latin caput and its Germanic relatives. One may suspect that this is another case of a 'container' word which acquired the meaning 'head' early in its history. As opposed to Tocharian A, Tocharian B exhibits a unique lexical innovation, äśce 'head'. The word is etymologically opaque; attempts to connect it with Indo-European words meaning 'ear of a cereal, sharp end' or 'bone' are mostly guesswork.

Finally, the Anatolian languages have their own 'head' words. Luwian harmaha/i- does not seem to have any known relatives. Hittite haršar/haršanaš shows a heteroclitic declension in which at least the oblique stem is strongly reminiscent of Vedic śírșnás $<* k_{0}^{\prime} h_{2 s n e ́ s} ;{ }^{15}$ but since neuters in ${ }^{*}-s r{ }^{*}{ }_{-s}(e) n$ were a distinct type in early IE, ${ }^{16}$ the similarity may well be accidental; at any rate, the initial $h$ in Hittite cannot be a regular reflex of $* \dot{k}$. We are therefore dealing with an Anatolian derivative of a familiar IE type, but the root remains to be identified. If the formation is deverbal, ${ }^{*} h_{3} \mathrm{Or}$ - 'rise, soar' is a plausible candidate. ${ }^{17}$ The verbal noun $* h_{3}$ ó $r-s_{0} / * h_{3 r} r-s(e) n$ - would mean something like 'elevation', hence 'upper part, top' and finally 'head' (an abstract evolving into a concrete noun).

Needless to say, nearly all the 'head' words discussed above have a large number of secondary senses such as 'person', 'physical life', 'the highest point, peak', 'starting-point, source', 'leader, chief', etc. Modern English head is quite typical in this respect.

\section{4. "Third generation" innovations}

As we have seen, the oldest reconstructible term for 'head' did not survive long in most of the lineages making up the IE family. It was soon replaced by synon-

\footnotetext{
${ }^{15}$ And a form like *kr ${ }^{\prime} h_{2}$ sér (loc. sg. 'in the head') must be posited as the basis for the derivation of *kerh ${ }_{2}$ srom $>$ Lat. cerebrum.

${ }^{16}$ As one of the anonymous reviewers points out, few if any such neuters can be confidently reconstructed on strictly comparative grounds. Nevertheless, the type must have existed in the protolanguage and enjoyed bursts of productivity in some of the daughter languages - not only in Anatolian, where the -eššar/-ešn- nouns preserve their heteroclisy; cf. the Vedic infinitives in -sani (fossilised locatives of $*_{-s r} / *_{-s}(e) n$ - neuters) and the related Greek ones in -ein $<*_{-}$ehen $<*_{-}$-sen.

${ }^{17} \mathrm{Cf}$. Ancient Greek óros 'mountain, highland' <*h3ór-es- (a neuter $s$-stem).
} 
ymous innovations everywhere except in Indo-Iranian, where it survived in a slightly modified shape. Neither did the branch-specific 'head' words prove to be as stable as "basic" lexemes could be expected to be. Semantic and lexical changes brought a new wave of 'head' words replacing their historical predecessors. These new lexical shifts have happened in historical times, so the innovations involved are usually of known origin, and their spread can sometimes be documented with textual evidence. It would be difficult to list all of them, so we shall focus on a few representative cases.

German Kopf began to replace the inherited Germanic word for 'head' in late Middle High German, about 1300. It comes from Vulgar Latin *coppa (cf. Italian coppa) < Late Latin cuppa 'cup' (borrowed into Old English as cuppe). The original meaning in High German was 'cup, chalice, drinking vessel'; already at an early date (ca. 1100) we can find the word applied to parts of the skull - the occiput or the brainpan. By the end of the Middle High German period kopf already functioned as a synonym of houbet 'head' (human or animal); the latter being increasingly restricted to formal or literary usage. Today, Kopf is the normal word for 'head', while Haupt survives as an antiquated word in some figurative senses ('the chief member of a group') and fixed expressions (see Siahaan 2011: 99-101, 104-105). Its most important remaining function is that of the first member of compounds, where it means 'main, chief, primary'. Having almost entirely lost its independent lexical status, Haupt- has thus evolved into a highly productive intensifying prefix, expressing importance. Note that Dutch kop, which has the same etymology, still has the core sense of 'cup', but can also refer to the head of an animal or - informally and often pejoratively - to a human head. It has not ousted hoofd 'head' but functions as its stylistically marked synonym in certain uses.

In the Romance group words for 'head' often go back to Latin caput (via Vulgar Latin * capu or the derivative *apetia). Forms descended from testa 'broken piece of earthenware, potsherd, tile' or 'earthen pot, urn' have successfully competed with them in several languages (French, Standard Italian), while in others they have related but less "basic" meanings, such as Romanian țeastă 'skull' or Portuguese testa 'forehead'. Where the reflex of testa has acquired the meaning 'head', as is the case with Italian testa and French tête, the reflex of caput loses its historical core sense but retains some of the secondary, figurative ones. Thus, both French chef and Italian capo can mean 'person in charge, commander, boss, chief'. ${ }^{18}$ The literal meaning 'head' may be fossilised in fixed

\footnotetext{
${ }^{18}$ Italian capo is more polysemous of the two; it can also mean 'top, beginning or end, item' etc.
} 
idioms, such as French de son (propre) chef'on one's own initiative' or Italian da capo a piedi 'from head to foot', but apart from such contextual uses the lexical shift is complete. The first stage of the process can be traced back to the fourth century: Late Latin testa is first documented with the meaning 'skull' in an epigram by Ausonius (who was active in Aquitaine). Tenth-century glosses and slightly later literary texts demonstrate that Old French teste was already used with the meaning 'head'. ${ }^{19}$ Note that, unlike cuppa, testa did not mean 'drinking vessel'; it could refer to any earthenware shell, often a broken pot or its fragment.

The modern reflexes of Proto-Slavic *golva still have the core meaning of 'head' (as a body part) everywhere in Slavic and do not show any symptoms of the semantic weakening that paves the way for lexical replacement. At the same time, however, the 'head' words in Slavic are rich in secondary senses, and some of their derivatives have already drifted away from their etymological meaning, losing all anatomical connotations. Thus, adjectives descended from Proto-Slavic * golvbnz (Polish główny, Czech hlavní, Russian glávnyj ${ }^{20}$ etc.) no longer mean 'pertaining to the head', but 'main, chief'. The Slavic word *lı $b$, with the original meaning 'skull', has shifted semantically to 'forehead, brow' in East Slavic (Russian lob) and to 'animal head' or 'human head' (facetiously or pejoratively) in Polish. The development is by no means recent; Polish $t e b$ has been a stylistically marked synonym of glowa at least since the 14th century, and it has developed its own family of idiomatic expressions. This is roughly the stage reached by kop in Modern Dutch or by kopf in late Middle High German, with one difference: the old meaning 'skull' is completely obsolete in Polish. The diminutive tebek $\sim$ lepek has acquired secondary senses parallel to those of glówka 'small head' (the diminutive of glowa); both words can refer to the "head" of a pin or a match, for example.

Needless to say, lexical innovations meaning 'head' can still arise in living languages. Suffice it to mention English noggin (originally a small drinking vessel), noddle (originally 'nape'), bean, nut, etc. Most of the time they remain restricted to the informal register, but their very presence makes lexical shifts possible. After all, most of the historically known successful competitors for the status of the basic 'head' word have had similar beginnings.

\footnotetext{
${ }^{19}$ Cf. Cravens (1982) for an attempt to reconstruct the semantic evolution of testa in early Romance.

${ }^{20}$ Borrowed from Church Slavonic.
} 


\section{Common patterns of semantic change and embodiment theory}

Whatever their origin, words for 'head' readily develop new uses, producing metonymic, synecdochic or metaphorical extensions of their core sense. Some figurative developments involving the notion of 'head' are cross-linguistically common despite language-specific preferences. ${ }^{21}$ The head is the topmost part of the human body; it can metonymically stand for a person, since we rely on traits such as facial features and hair colour to recognise fellow humans. Straightforward analogy between the longitudinal orientation of the human (or more generally animal) body and inanimate objects inspires such secondary senses as HEAD = PEAK, FRONT PART, WORKING END (of a tool) etc. "Orientational metaphors" (Lakoff and Johnson 1980: 14-21) generate associations between the notion of 'head' and control, importance and high status; hence HEAD $=$ MOST IMPORTANT MEMBER of a hierarchy (the leader, mastermind, person in charge). These associations are reinforced by the fact that the head is a vitally important body part, independently of its topmost position (therefore, additionally, HEAD = LIFE). But the head is also regarded as the locus of the mind, thought and intelligence, hence more complex conceptual metaphors involving the image of HEAD as a CONTAINER for thoughts, ideas, memories, plans, visions, etc. These do not necessarily produce new senses, but are responsible for innumerable set phrases, idioms and figurative expressions in which mental processes are conceptualised as physical entities located in the head (as well as metaphorically entering and leaving it).

Not all languages extend the meaning of HEAD in the same way, but in the IE family, in particular, 'head' words tend towards extreme polysemy. The entry "head, $n$. " in the OED (as updated in June 2013) lists no fewer than 56 senses of the word, many of them with numerous subsenses; they are followed in the dictionary by a long list of set phrases in which head is a keyword, and dozens of compounds with head as the first member. Even if several of those 56 senses are rare, dialectal, obsolete or hermetically technical, their sheer number testifies to the importance of HEAD in shaping English-language metaphorical mappings. Much the same is true of other Indo-European languages: a plethora of secondary senses and conceptualisations quickly grows round the basic word for 'head'. This development may be followed by the marginalisation and eventual loss of the "literal" anatomical meaning, which is taken over by a new lexical competitor, whereupon the cycle can start over again.

\footnotetext{
${ }^{21} \mathrm{Cf}$. the contributions by Siahaan and Aksan in the Maalej and Yu (2011) volume.
} 
This scenario has been enacted repeatedly since the earliest stages in the history of the Indo-European family. Other common words for body parts (except those meaning 'hand') do not show this kind of explosive semantic evolution accompanied by lexical shifts. They are highly conserved, which is what we could predict from their high frequency of use and the tendencies observed by Pagel, Atkinson and Meade (2006). However, as shown by Vejdemo and Hörberg (2016), semantic factors may override frequency effects. We can therefore conclude that the concept HEAD was not merely a cognitive representation of a body part in PIE and its descendants. It played a central role in conceptualisations based on linguistically manifested embodiment. To use the terminology of Sharifian et al. (2008: 3-4), the IE languages were already at an early date "cephalocentric" (or "head-centred"): the head was considered to be the locus of the mind, intelligence and personality, and therefore the "real centre" of the human body, with all the consequences for embodied cognition and semiosis. Since the IE languages of today still belong to the same type, such a conceptualisation may seem self-evident to their speakers, but it is not. The same role may be played by another "central" organ or body part - usually the heart or the abdomen - in some cultures.

Conceptualisations can work in the reverse: if the head is thought of as a container for ideas, that facilitates semantic change in words originally meaning 'container', 'shell' etc., which may be co-opted as stylistically marked synonyms of the main 'head' word. If the topmost position of the head is emphasised in productive metaphors and set idioms, a word with the original meaning of 'peak, summit' may also come to be used with reference to the head. Etymology suggests that this indeed has happened many times in the history of IndoEuropean. There may be intermediate stages: in several of the cases analysed above a word meaning 'cup, vessel' or the like first developed an indirect association with 'head', acquiring the meaning 'skull, cranium', i.e. the hard encasing of the brain, which physically resembles a piece of pottery. ${ }^{22}$ Also in those cases where the origin of a particular 'head' word is disputable, insights based on the cases when such words are provided by "reversed conceptualisation" may be useful. Thus the "container" etymology of Latin caput and its Germanic cognates or the "peak" etymology of Hittite haršar/haršanaš are attractive not only because they make sense formally but because they fit into a familiar pattern attested elsewhere.

\footnotetext{
${ }^{22}$ Note that skull-cups could actually be crafted from human crania. Cf. Sanskrit kapála- (perhaps a root-cognate of Latin caput), which means 'cup, bowl' or 'skull, cranium', and in some Hindu and Buddhist traditions refers to a ritual skull-cup.
} 
Far be it from me to claim that this "cephalocentrism" and its effect on the semantic evolution of words for 'head' is a unique property of the IE languages and cultures. Nevertheless, Holman et al.'s (2008) results (see Section 1 above), suggest that IE really stands out as having particularly unstable words for 'head' (in comparison with most other language families). As pointed out by one of the reviewers of this article, Proto-Semitic * $r a$ 's 'head' is widely attested throughout the Semitic family (Hebrew rōš, Arabic ra's, Ge'ez rə’s', Amharic ras, etc.), which implies its stability over long spans of time. ${ }^{23}$ At the same time, however, the Semitic languages show a wide range of secondary meanings similar to those found in IE ('top', 'promontory', 'leader, the most important person', etc.). Why is 'head' less prone to lexical replecement there? Possibly some crucial conceptualisations are rare or missing, for example HEAD $=$ CONTAINER (for thoughts, ideas, plans, etc.) - the association which has enabled words with meanings like 'pot, vessel' to replace 'head' in IE several times independently. ${ }^{24}$

It should also be noted that in Akkadian (East Semitic) the inherited word for 'head', $r \bar{e} s ̌ u$, is usually attested in figurative meanings such as 'top', 'beginning', 'first rank or quality' or 'servant, slave', whereas the literal meaning 'head' is taken over by qaqqadu, whose Hebrew and Ugaritic cognates mean 'skull'. In fact, from the Old Akkadian period onwards qaqqadu continued to replace $r \bar{s} s ̌ u$ also in its metonymic and metaphorical uses: it could mean 'person', 'leader', 'head tax', 'top', 'start, beginning', 'working end (of a tool)', etc. This encroachment is closely analogous to semantic processes observed in several IE languages. ${ }^{25}$

\footnotetext{
${ }^{23}$ It has been replaced in most South Ethiopic languages either by a loanword (Arabic $\operatorname{dim}_{\bar{a}} \bar{y}^{-}$ 'brain', with a shift of meaning) or by reflexes of *gunnän, of uncertain origin, cf. Kogan (2011: 220). It is therefore slightly less stable than, for example, * 'ayn- 'eye' (truly pan-Semitic) or *šinn'tooth' (replaced in a few isolated cases). On the situation in Akkadian, see below.

${ }^{24}$ Whether this conceptualisation is really less widespread in Semitic than in IE is a question I do not feel competent to answer. I must leave my tentative explanation to be evaluated by experts on the Semitic languages.

${ }^{25}$ Interestingly, another word affected in this way is $i d u$, the Akkadian reflex of Proto-Semitic *yad- 'hand' (extremely well conserved throughout the rest of the family). In Akkadian, it may have the anatomical sense of 'arm' or 'wing', but is mostly attested in idiomatic or figurative meanings like 'span, border', 'handle', 'fathom' or 'strength'. In the meaning 'hand' we find Akkadian $q \bar{a} t u$, an innovation of obscure origin, which moreover displays a wide range of secondary senses, often with metaphorical reference to power, control, authority, possession or jurisdiction.
} 


\section{Conclusion}

Rates of lexical replacement are believed to depend mainly on the frequency of use: in the first approximation, high-frequency words survive more successfully than rare ones. The frequency effect is stochastic rather than deterministic - that is, we define it in terms of probability. Individual words may deviate from the overall statistical tendency simply because the process involves a good deal of random variation. However, some of the words expressing "basic meanings" display wayward behaviour so often that we are justified in looking for other effects that may be strong enough to override frequency. The claim made above is that cultural and semantic factors (including culture-specific embodied cognition) may accelerate lexical evolution by helping words to develop new functions and to acquire new synonyms. To quote Vejdemo and Hörberg (2016: 4),

[i]t is also very likely that cultural considerations are very important for which words get replaced, and that these cultural considerations vary between speaker communities, and over time. In addition, specific semantic domains (such as body parts, kinship terms, colors etc.) probably have domain specific [sic] tendencies when it comes to likelihood of lexical replacement. Our investigation of lexical replacement takes none of this into account, and instead tries to investigate if it is possible to find evidence for domain-overriding generalizations of which factors can affect the rate of lexical replacement.

In this paper, we argue that "embodied cognition" has a similar effect. In IndoEuropean languages (but not necessarily in other language families), words for 'head' evolve semantically faster and are replaced by competing synonyms more often than could be expected from their very high frequency of use and empirically confirmed stability across language families. A similar argument could be made for the concept of HAND (or ARM-AND-HAND) as opposed to TOOTH, FOOT, LIVER, HEART, EYE, EAR, etc. This suggests that metaphorical thinking in Indo-European cultural traditions has for a long time (measured in thousands of years) been "cephalocentric", and possibly also "chirocentric". Embodiment theory offers an elegant explanation of this bias. 


\section{References}

Aksan, M. 2011. "The apocalypse happens when the feet take the position of the head: Figurative uses of 'head' and 'feet' in Turkish". In: Maalej and Yu (eds.), 241-256. Andersen, E.S. 1978. "Lexical universals of body-part terminology”. In: Greenberg, J.H. (ed.), Universals of human language (Vol. 3: Word structure). Stanford, CA: Stanford University Press. 335-368.

Blust, R. [2009] 2013. The Austronesian languages (revised edition). Canberra: AsiaPacific Linguistics Research School of Pacific and Asian Studies, The Australian National University.

Brown, C.H. 1976. "General principles of human anatomical partonomy and speculations on the growth of partonomic nomenclature". American Ethnologist 3(3). 400 424.

Buck, C.D. 1949. A dictionary of selected synonyms in the principal Indo-European languages: A contribution to the history of ideas. Chicago-London: The University of Chicago Press.

Cravens, T.D. 1982. "Cross-language evidence in etymology: The origin of 'testa' as 'head' in Romance". Neuphilologische Mitteilungen 83(1). 53-60.

Enfield, N.J. 2006. "Elicitation guide on parts of the body". Language Sciences 28. 148157.

Holman, E.W., S. Wichmann, C.H. Brown, V. Velupillai, A. Müller and D. Bakker. 2008. "Explorations in automated language classification". Folia Linguistica 42(2). 331-354.

Goddard, C. 2001. "Lexico-semantic universals: A critical overview". Linguistic Typology 5(1). 1-65.

Kogan, L. 2011. "Proto-Semitic lexicon”. In: Weninger, S. (ed.), The Semitic languages: An international handbook. Berlin-Boston: Walter de Gruyter. 179-258.

Lakoff, G. and M. Johnson. 1980. Metaphors we live by. Chicago-London: University of Chicago Press.

Maalej, Z.A. and N. Yu (eds.). 2011. Embodiment via body parts: Studies from various languages and cultures. Amsterdam-Philadelphia: John Benjamins.

Nussbaum, A. 1986. Head and horn in Indo-European. Berlin-New York: Walter de Gruyter.

OED = Oxford English Dictionary Online. December 2016. Oxford University Press.

Pagel, M., Q. Atkinson and A. Meade. 2007. "Frequency of word-use predicts rates of lexical evolution throughout Indo-European history". Nature 449. 717-720.

Sharifian, F., R. Dirven, N. Yu and S. Niemeier (eds.). 2008. Culture, body, and language: Conceptualizations of internal body organs across cultures and languages. Berlin: Walter de Gruyter.

Siahaan, P. 2011. "HEAD and EYE in German and Indonesian figurative uses". In: Maalej and $\mathrm{Yu}$ (eds.), 93-116.

Vejdemo, S. and T. Hörberg. 2016. "Semantic factors predict the rate of lexical replacement of content words". PLOS ONE 11(1): e0147924. doi:10.1371/journal.pone.0147924. 
Address correspondence to:

Piotr Gąsiorowski

Faculty of English

Adam Mickiewicz University in Poznań

al. Niepodległości 4

61-874 Poznań

Poland

gpiotr@wa.amu.edu.pl 